\section{Ectopic Thymoma- Presenting as a Large Pleural Mass}

Neerja Gulati*, Frances Schmidt, Setu Patolia, Dharani Narendra, Muhammed Perwaiz, Danilo Enriquez, Joseph Quist and Joseph Geradith Interfaith Medical Center, Brooklyn, NY, USA

\begin{abstract}
Thymoma is the most common neoplasm of the anterior mediastinum. Patients may be asymptomatic or present with symptoms of local compression, myasthenia gravis or other paraneoplastic syndrome. We present case of a middle aged woman with a large pleural based mass who was relatively asymptomatic Biopsy of the mass revealed thymoma type AB. Work up showed that she has multiple abdominal masses etiology of which is not known. Whenever feasible, surgery is the treatment of choice for thymoma.Our patient is undergoing chemotherapy with the goal that once the tumor size is reduced she will be able to undergo surgery.
\end{abstract}

Keywords: Thymoma; Mediastinum; Pleural

\section{Case Report}

59 year female with no significant past medical history was referred by her primary care physician for abnormal CXR. She had chronic cough with small mucoid expectoration for last 5-6 months. There was no history of hemoptysis/fever/weight loss or night sweat. She was born in Haiti, had positive Purified Protein Derivative (PPD) and Chest $\mathrm{x}$-ray (CXR) done 2 years back was negative.

She was working as a health aid and denied history of exposure to patient with active Tuberculosis (TB). There were no allergies. She had never smoked and was not on any medications. There was history of uterine fibroids for which she was suggested surgery in the past but she refused. She was post-menopausal.

On examination she was thin built (BMI 17.4).Her vitals were stable with oxygen saturation on room air of $96-97 \%$. There was no significant lymphadenopathy or thyromegaly. On chest examination there was decreased air entry at right base. Cardiovascular examination was normal. On abdominal palpation, there was a big firm, non-tender pelvic mass reaching up to umbilicus.

Initial labs were essentially normal except for mild normocytic normochromic anemia.

Chest X-ray showed severe elevation of right hemi diaphragm with a mass occupying almost the entire right hemi thorax (Figure 1).

CT scan chest revealed a large mass occupying majority of the right hemithorax compressing right main stem bronchus - origin of the mass was uncertain and was associated with atelectatic lung (Figures 2-6).

CT scan of abdomen \& pelvis showed large suprahepatic mass and a very large pelvic mass. Spleen appeared to be heterogenous.Additional mesenteric masses were noted (Figures 7 and 8).

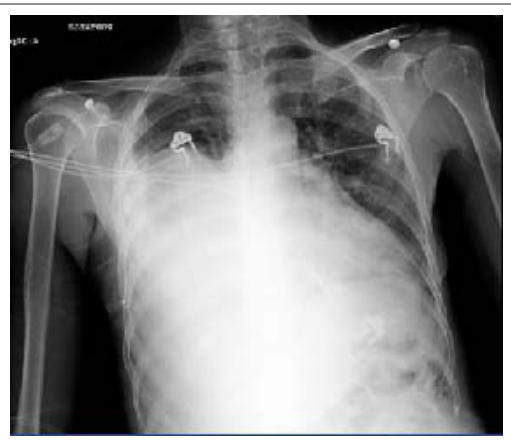

Figure 1: CXR PA view showing large mass in right hemi thorax.

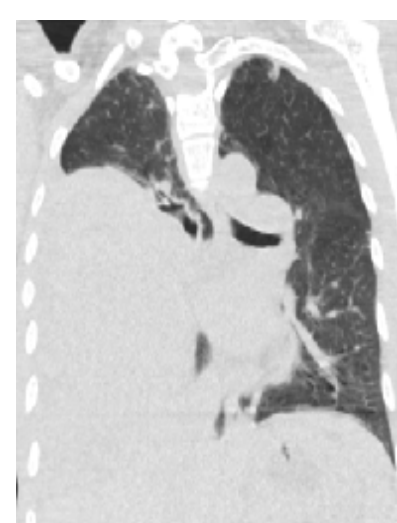

Figure 2: CT scan showing mass in right hemi thorax compressing right main bronchus and associated atelectasis of lung.

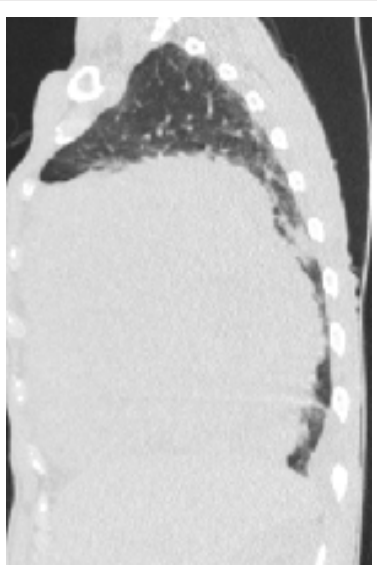

Figure 3: CT scan showing mass in right hemi thorax compressing right main bronchus and associated atelectasis of lung.

${ }^{*}$ Corresponding author: Neerja Gulati, Department of Medicine, Interfaith Medical Center, NY, USA, Tel: 718-613-4000; Fax: 718-613-4893; Email: gulati_neerja@yahoo.com

Received September 05, 2012; Accepted September 26, 2012; Published September 28, 2012

Citation: Gulati N, Schmidt F, Patolia S, Narendra D, Perwaiz M, et al. (2012) Ectopic Thymoma- Presenting as a Large Pleural Mass. J Pulmon Resp Med 2:131. doi:10.4172/2161-105X.1000131

Copyright: $\odot 2012$ Gulati N, et al. This is an open-access article distributed under the terms of the Creative Commons Attribution License, which permits unrestricted use, distribution, and reproduction in any medium, provided the original author and source are credited. 
Patient underwent CT guided percutaneous biopsy of the mass in right hemi thorax. Tumor composed of small undifferentiated cells with no obvious glandular or squamous differentiation. Immunohistochemical staining showed: positive CAM 5.2,rare CK7,P63,CK5/6,CD5,calretinin,Ki-67,TdT,CD4 and CD8, and negative NSE,synaptophysin,chromograninA,CD20,CD3.- consistent with thymoma, favor type AB likely pleural origin (Figures 9-13).

As thymoma has strong association with myasthenia and other systemic syndromes, she was reviewed again for any muscular weakness. Serum protein electrophoresis, immunofixation, quantitative

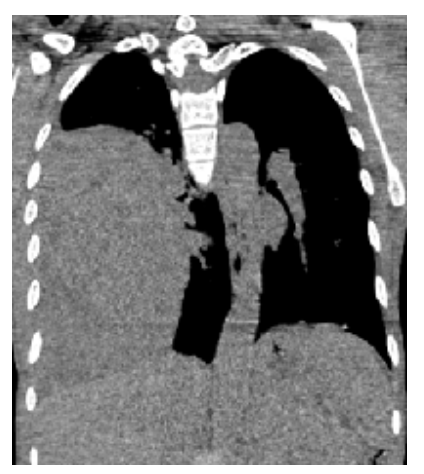

Figure 4: $\mathrm{CT}$ scan showing mass in right hemi thorax compressing right main bronchus and associated atelectasis of lung.

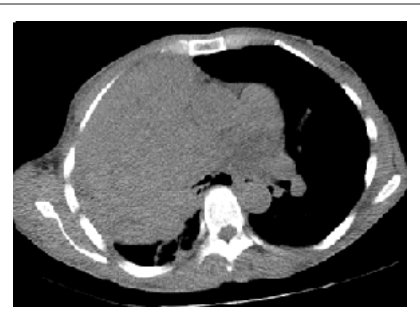

Figure 5: Large heterogeneous mass in right hemi thorax encroaching mediastinum.

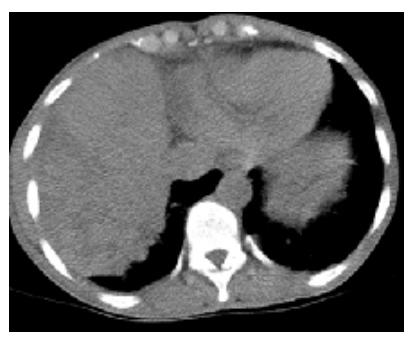

Figure6: Large heterogeneous mass in right hemi thorax encroaching mediastinum.

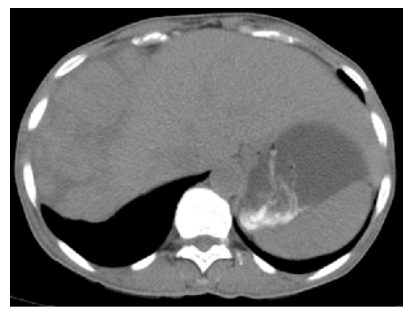

Figure 7: CT scan abdomen with oral contrast showing heterogeneous mass adjacent to liver.

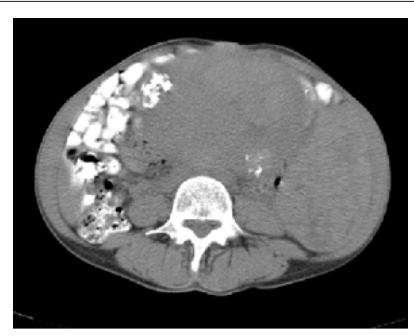

Figure 8: CT scan pelvis with oral contrast showing large pelvic mass pushing the bowel loops and also an additional mass on left side.

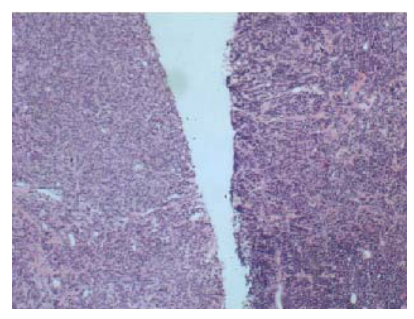

Figure 9: H\&E stain.

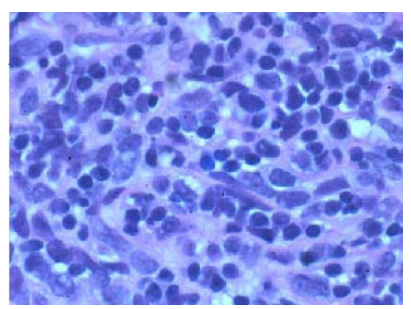

Figure 10: H\&E stain.

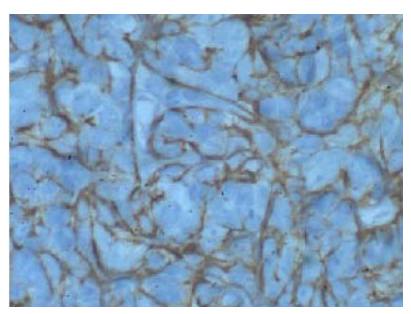

Figure 11: CK5/6 stain.

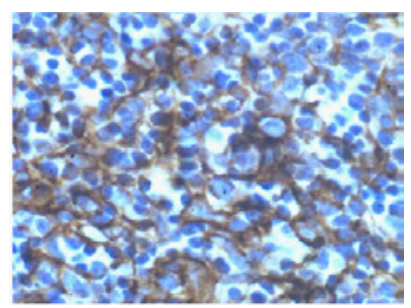

Figure 12: CAM 5.2 stain.

immunoglobulin were normal. On PET scan, lung mass had SUV of 4.7 and pelvic mass had SUV of 2.8 .

Patient was assessed as a case of advanced thymoma (likely stage IVa but at least stage III) likely pleural origin. She was referred to oncologist for chemotherapy, expecting that chemotherapy will shrink the thoracic mass and tumor may be amenable to surgery afterwards. 


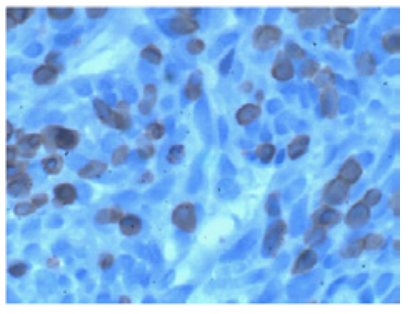

Figure 13: CD3 stain.

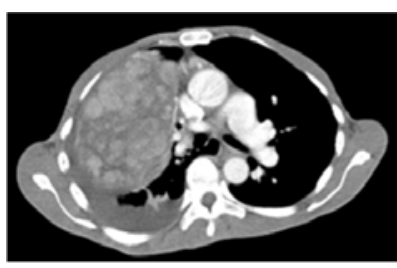

Figure 14: CT scan chest with contrast showing increased heterogeneity of right thoracic mass and pleural- effusion on right side.

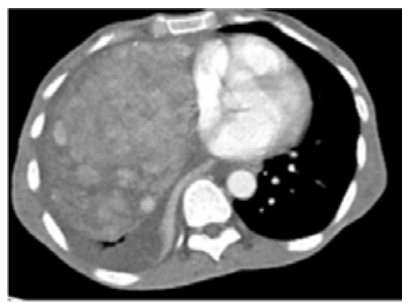

Figure 15: CT scan chest with contrast showing increased heterogeneity of right thoracic mass and pleural- effusion on right side.

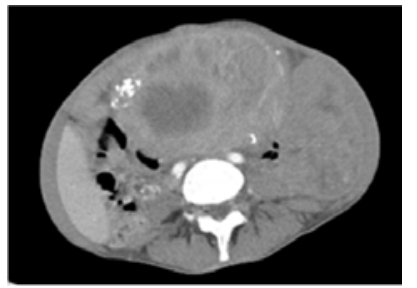

Figure 16: CT scan pelvis with large heterogeneous pelvic masses.

The etiology of abdominal masses was not clear. Patient refused the biopsy of abdominal masses.

She was started on neoadjuvant chemotherapy (cisplatin and etoposide). Over next 3 months her there was no significant change in her clinical condition except for nausea and vomiting after chemotherapy. She underwent repeat CT chest and abdomen which revealed no significant change in the size of thoracic and pelvic masses but there was new appearance of right sided pleural effusion and heterogeneity in thoracic mass (Figures 14-16).

Since there was no clinical/radiologic improvement her chemotherapy regimen was changed to cisplatin, Adriamycin and cyclophosphamide. She will be reassessed after 6 cycles if she can undergo surgery. If the abdominal masses do not regress in size, patient will be counseled again for biopsy of the abdominal mass.

\section{Discussion}

The thymus gland is located behind the sternum in front of the great vessels; it reaches its maximum weight at puberty and undergoes involution thereafter. In early life, the thymus is responsible for the development and maturation of cell-mediated immunological functions. The thymus is composed predominantly of epithelial cells and lymphocytes. Precursor cells migrate to the thymus and differentiate into lymphocytes. Most of these lymphocytes are destroyed, with the remainder of these cells migrating to tissues to become T lymphocytes.

Thymoma, the most common neoplasm of the anterior mediastinum, originates within the epithelial cells of the thymus. It accounts for $20-25 \%$ of all mediastinal tumors and $50 \%$ of anterior mediastinal masses. The etiology of thymoma is not clear; however, it has been associated with various systemic syndromes. Most patients are between $40-60$ years of age. There is slight male predominance.

Of patients with a thymoma, one third to one half are asymptomatic .One third of cases are found incidentally on radiographic examinations during a workup for MG.

Patient may present with local symptoms related to the tumor encroaching on surrounding structures like cough, chest pain, superior vena cava syndrome, dysphagia, and hoarseness of voice. $30-40 \%$ of patients with thymoma experience symptoms of myasthenia gravis. An additional $5 \%$ of patients have other systemic syndromes including red cell aplasia, dermatomyositis, systemic lupus erythematous, Cushing syndrome and syndrome of inappropriate antidiuretic hormone secretion.

Postero Anterior (PA) and lateral chest radiographs can detect most thymomas. On the PA view, the lesion typically appears as a smooth mass in the upper half of the chest. The mass usually projects predominantly into one of the hemi thoraces. Computed Tomography (CT) scanning may delineate the mass further or detect a smaller tumor missed on radiograph. CT scanning with intravenous contrast dye is preferred to show the relationship between the thymoma and surrounding vascular structures to define the degree of its vascularity and to guide the surgeon in removal of a large tumor, possibly involving other mediastinal structures. PET scan helps in staging and in excluding extramediastinal involvement.

Diagnosis is made by tissue biopsy. Sample can be obtained by CT guided core biopsy, mediastinoscopy, VATS or open surgical biopsy depending upon location of the tumor.

There is continuum of differentiation from thymoma to thymic carcinoma. Carcinoma and thymoma can exist synchronously. Heterogeneity is common within thymomas and cellular composition correlates with biological behavior of tumor.

\section{Treatment}

Due to rarity of the tumor, there are no randomized controlled trials. Complete surgical excision is the preferred treatment approach whenever technically feasible. Thymomas are chemotherapy sensitive. Chemotherapy before and/or after surgery and radiation therapy may be useful in appropriately selected patients.

Stage I- Complete surgical removal of the tumor

Stage II- Complete surgical removal of the tumor +/- Radiation after surgery

Stage III and IV and Recurrent Disease- Complete surgical removal of the tumor, if possible radiation after surgery (Table 1).

Radiation alone if the tumor is considered unresectable (unable to 


\begin{tabular}{|l|l|}
\hline Stage & Features \\
\hline I & $\begin{array}{l}\text { Macroscopically encapsulated with no microscopically detectable } \\
\text { capsular invasion }\end{array}$ \\
\hline II & $\begin{array}{l}\text { Macroscopic invasion of mediastinal fatty tissue or mediastinal pleura, or } \\
\text { microscopic invasion into the capsule }\end{array}$ \\
\hline III & $\begin{array}{l}\text { Macroscopic invasion into surrounding structures(pericardium, great } \\
\text { vessels, lung) }\end{array}$ \\
\hline IVA & Pleura or pericardial dissemination \\
\hline IVB & Lymphogenous or hematogenous metastases \\
\hline
\end{tabular}

Table 1: Masaoka Staging System.

be completely removed). Chemotherapy before and/or after surgery and/or radiation, especially if the tumor is considered unresectable before surgery as in this patient.

Induction therapy for locally advanced thymic tumor with cisplatin, etoposide, radiation is well tolerated. Many patients show partial radiographic response. The majority of patients can undergo a complete resection with treatment.

In a series of 21 patients with unresectable malignant thymoma, patients underwent multidisciplinary approach with induction CT, followed by surgical resection, RT and consolidation therapy. The seven-year disease-free and overall survival rates were 77 and 79 percent, respectively. In another series of 30 patients with stage III and IVA who underwent neoadjuvant CT, surgery and post-op RT; $10 \mathrm{yr}$ survival rates were 85.7 and $76.1 \%$ respectively.

Chemotherapy alone is the treatment if the cancer has spread widely throughout the body and there are no significant symptoms associated with the tumor in the chest. No large randomized trials have been conducted to guide the choice of treatment because of the rarity of the disease. Single agents with established activity include cisplatin, ifosfamide and corticosteroids. Combination chemotherapy generally produces higher response rates than single agents. Various treatment regimens that have been used are-

Cisplatin, doxorubicin and cyclophosphamide (PAC), with or without prednisone
Cisplatin, doxorubicin, cyclophosphamide and vincristine (ADOC)

Etoposide and cisplatin (EP)

Etoposide, ifosfamide and cisplatin (VIP)

Thymic tumors that express somatostatin receptors may have clinically meaningful responses to octreotide with or without prednisone.

Small observational series and case reports indicate that receptor tyrosine kinase inhibitors may have clinically relevant activity in patients with metastatic thymic carcinoma.

The single most important factor predicting the outcome of patients with thymomas is evidence of invasion. Relapse after primary therapy for a thymoma may occur after 10-20 years. In a series of 126 patients who underwent complete resection of a thymoma, 24 eventually recurred. The time to recurrence ranged from 4 to 175 months (mean 68 months). The initial sites of recurrence were pleural, local, or distant in 22,6 , and 5 cases respectively.

Thymomas have been associated with the development of second malignancies. In a review of the Surveillance, Epidemiology, and End Results (SEER) database of thymoma cases in the United States (1973-1988), 849 cases were identified, of which 66 were found to have second malignancies. Therefore, long-term follow-up probably should continue to be performed throughout the patient's life.

In our patient the clinical presentation was atypical and there was no thymic enlargement or mediastinal mass seen on radiology. The tumor was described as a pleural thymoma as it seems to originate from the pleura although on pathology pleural involvement was not described. Cases have been previously reported with the tumor originating from ectopic thymic tissue in the pleura.

The tumor in our patient is at least stage IIIa or possibly stage IVa. The pelvic mass is likely a fibroid uterus but the nature of the abdominal masses remain uncertain. The treatment will remain combination of chemo and or RT pre or post surgery if possible. 